\title{
Optimization of elstomeric micro-fluidic valve dimensions using non-linear finite element methods
}

\author{
H. A. Khawaja', I. Raouf ${ }^{2}$, K. Parvez ${ }^{3}$ and A. Scherer ${ }^{4}$ \\ ${ }^{1}$ MPhil Student, Department of Engineering, University of Cambridge, UK \\ hak23@cam.ac.uk \\ ${ }^{2} P h D$ Student, Electrical Engineering, California Institute of Technology, USA \\ imran@caltech.edu \\ ${ }^{3}$ Director of the Research Centre for Modeling \& Simulation, Professor of \\ Aerospace Engineering, National University of Sciences \& Technology, \\ Pakistan, khalid-rcms@nust.edu.pk \\ ${ }^{4}$ Director of the Kavli Nanoscience Institute, Neches Professor of \\ Electrical Engineering, Applied Physics and Physics, California Institute of \\ Technology, USA, etcher@caltech.edu
}

\begin{abstract}
We use a nonlinear finite element (FE) method model to compare, optimize and determine the limits for useful geometries of microfluidic valves in elastomer polydimethylsiloxane (PDMS). Simulations have been performed with the aim of finding the optimal shape, size and location of pressurization that minimizes the pressure required to operate the valve. One important constraint governing the design parameters is that the stresses should be within elastic limits, so that the component remains safe from any type of structural failure. To obtain reliable results, non-linear stress analysis was performed using the Mooney-Rivlin 9 parameter approximation which is based on the Hyper Elastic Material Model. A 20 noded brick element was used for the development of FE model. Mesh sensitivity analysis was also performed to assess the quality of the results. The simulations were performed with commercially available FE modeling software, developed by ANSYS Inc. to determine the effect of varying different geometric parameters on the performance of micro-fluidic valves.

The aim of this work is to determine the geometry of the channel crosssection that would result in the largest deflection for the least applied pressure, i.e. to minimize the pressure needed to operate the valve.
\end{abstract}

\section{INTRODUCTION}

The field of micro-fluidics lies at the interface between engineering, chemistry, and biology. This field has seen rapid growth as numerous applications have evolved in biology, chemistry, diagnostics and analysis for the control of picoliter volumes on chips. A common application is the development of lab-on-a-chip systems that could be used to explore single cell and single molecule biophysics, miniaturize chemical assays, reduce the quantities of reagent and enable massively-parallel, high-throughput biochemical 
analyses. The engineering challenges include optimizing the mixing, reaction, separation, pre-concentration, and detection of chemical species at the micro-scale [1].

Elastomers have evolved as some of the most suitable materials from which such lab on a chip systems can be constructed, as their low modulus of elasticity permits the miniaturization of valves. The microfluidic valve is a key component in many lab on chip systems, and valves of various designs have been fabricated. However, it is worth noting that the design of these valves has so far evolved mostly by experimentation. We believe that state of the art engineering design software can be reliably used to better understand, and optimize elastomer structures. Due to nonlinear behavior and complex geometries within practical microvalve structures, we could not find reliable macro-models or reduced order models for predicting the behavior of such structures.

Since it is extremely hard to find local stresses and strains in PDMS elastomer embedded structures, simulation-based design helps us to determine the mechanical design parameters, such as the region of operation and points of stresses which help in reliable designs.

Unger et al. [3] have discussed the fabrication of a micro-fluidic valve in a micro-fluidic chip constructed from Silicone RTV 615. Similarly, Kartalov et al. [5] have produced a (linear) model of micro-fluidic valves fabricated from elastomers. Lotters et al [6] have investigated the mechanical properties of the elastic polymer (PDMS) for its application in micro-fluidic chips. The optimization of these systems is complicated by the fact that the mechanical behavior of PDMS is non-linear [4], which must be taken into account when using Finite Element Analysis (FEA) techniques [2]. Other complexities of modeling elastomers are unusually large deformations (which introduces constraints on meshing) and nonlinear geometry.

This work is related to the optimization of the valve geometry within a microfluidic chip fabricated from an elastomer (PDMS). Finite element simulations have been performed with the aim of finding the optimal shape, size, and location of pressure application that minimizes the external pressure required to operate the valve. One important constraint is that stresses should be within elastic limits, so that the PDMS elastomer remains safe from any type of structural failure.

\section{OPERATION OF VALVE}

The PDMS valve is intended to be used for controlling of fluid flow within a sub-millimeter wide channel of dimension. Any controlling device, such as electromagnetic solenoids, pneumatic devices, mechanical actuators, or phase can be used. Moreover, it is not necessary that the valve should be closed completely in order to stop the flow fully, since, at this small scale, capillary effects are important. It is assumed that a reduction in the cross-sectional area of channel of $90 \%$ would be sufficient to stop the flow of fluid [7]. This also helps to avoid contact which occurs between valve surfaces. Contact has additional complexity for simulation since it introduces a severe non-linearity. Figure 1 shows the schematic diagram of typical micro-fluidic valve model geometry.

In Figure 1 we show a typical cross-sectional view of an open valve, with a semi-circular fluid channel. To close this valve, pressure is applied normal to the fluid flow direction and also normal to the surface of the channel. This deforms the channel and thereby constricts the flow. Appropriate deformation of the channel will stop the flow completely.

All layers of simulated valves are out of bulk material of same type. While building valves using separate layers with multi-layer soft lithography is also common [3], the valves simulated here can be produced by using molds produced by wax printers or other means. 


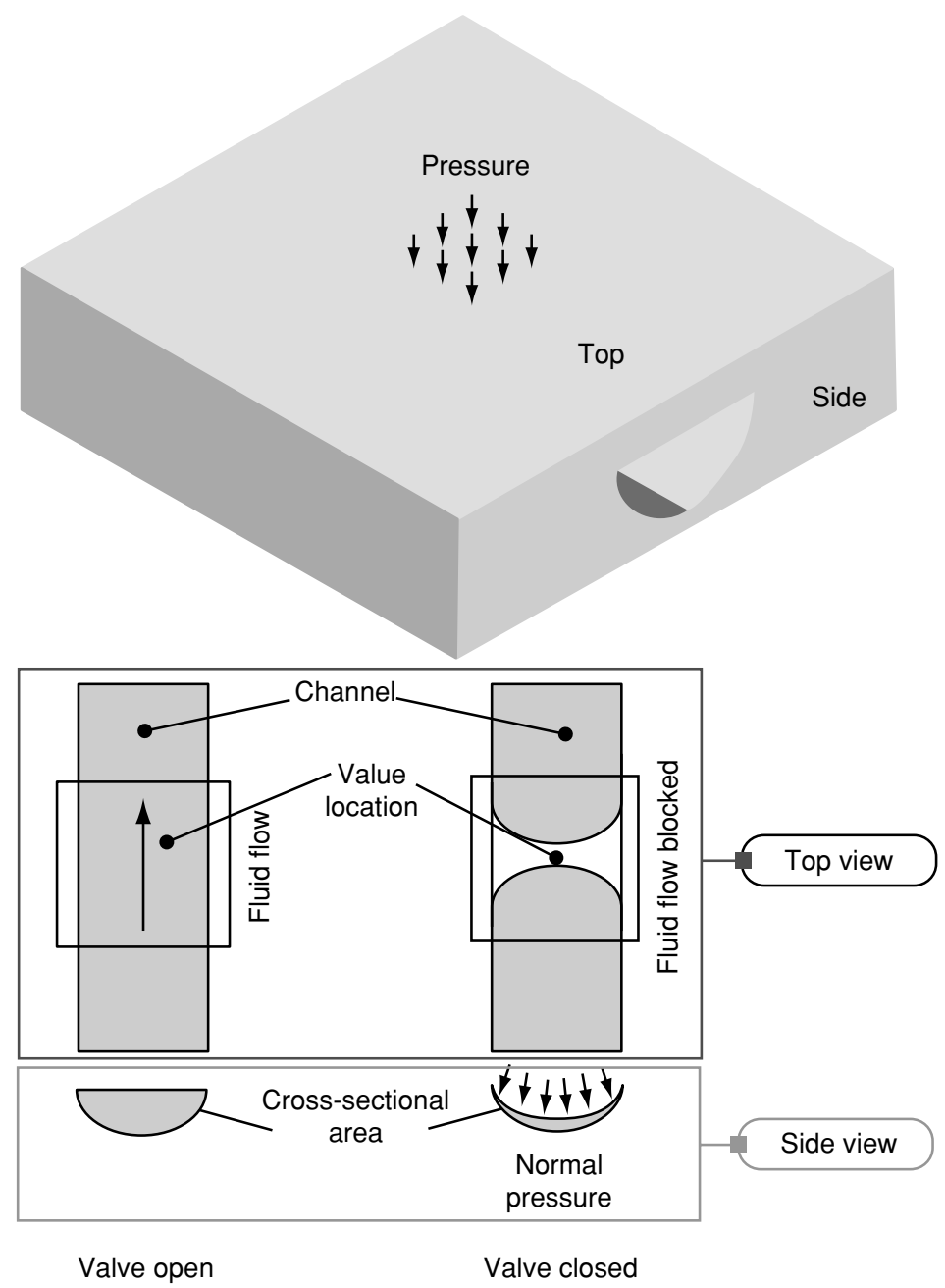

Figure 1 Schematic diagram of micro-fluidic valve.

Structures without layers avoid problems of delamination and alignment. Using injection molds also seems more promising in a production setting.

\section{FINITE ELEMENT ANALYSIS}

\subsection{HYPER ELASTIC MATERIAL MODEL}

The elastomeric polymer PDMS was selected for the fabrication of micro-fluidic channel due to its inertness to chemical reactions and its elastic behavior [7]. A typical Stress-strain behavior of PDMS is shown in figure 2. Also shown in figure 2 is the Non-Linear-HyperElastic material model with 9th order Mooney-Rivlin approximation $[9,11,12,13]$, which was used to approximate the behavior of the PDMS in our FE model [5].

A $9^{\text {th }}$ order approximation is accurate but requires considerable computational capabilities [9]. As the model developed was not very large in terms of number of nodes considering a 


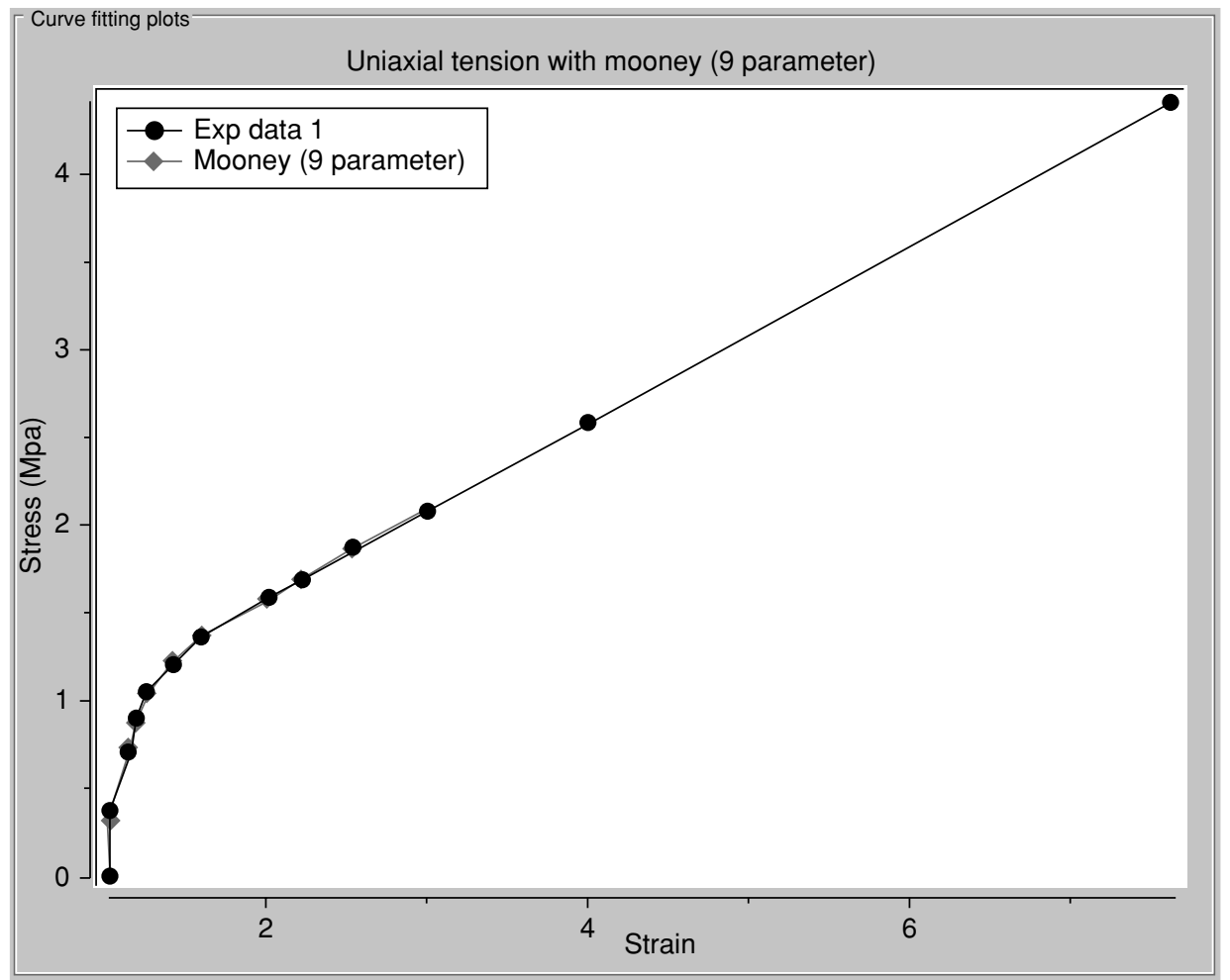

Figure 2 PDMS stress vs strain behavior and mooney-rivlin 9 parameters approximation.

higher order model was possible. In an actual fluidic chip, the valves are separated by appropriately large distances which allow this work to be directly applied to complex chips.

\subsection{LIMITATION IN HYPER ELASTIC MATERIAL MODELLING}

Most material models in commercially available finite element analysis codes allow the analyst to describe only a subset of the structural properties of elastomers [2]. These limitations are thoroughly discussed in the literature $[2,9]$. Based on the limitations involved in finite element hyper elastic material modeling it was assumed that dimensions considered are of pre-strained model. This does not affect experimental use. One can close the valve once or apply higher than valve closure pressure to enable pre-strained region for subsequent use.

\subsection{FINITE ELEMENT MESH}

In finite element analysis too fine a mesh is inefficient, whilst too course a mesh may give unreliable results. Solid 20 node 186 element was used for building finite element mesh. It was selected based on the recommendation in ANSYS technical manuals $[9,10]$.The mesh for the every model built was refined in order to identify the optimum mesh; the results of one of the mesh developed are shown in figure 3 and indicate that that optimized FE Model has 3645 elements.

In this analysis element skewness and elements shape after distortion were manually observed. Keeping in view the results FE mesh pattern was adjusted accordingly. One of the FE Mesh Pattern of valve model is shown in figure 4. 


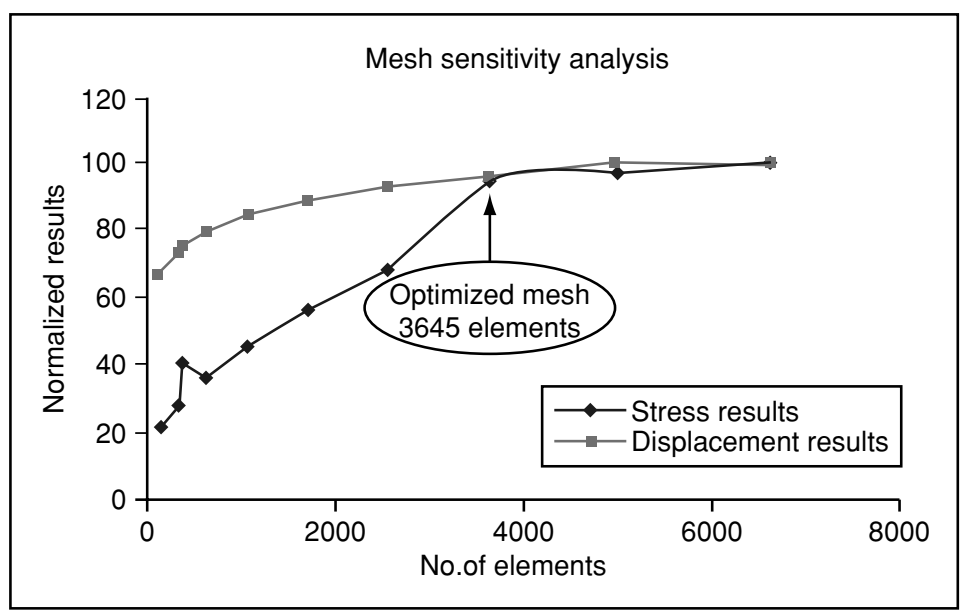

Figure 3 Variation in results obtained through mesh refinements.

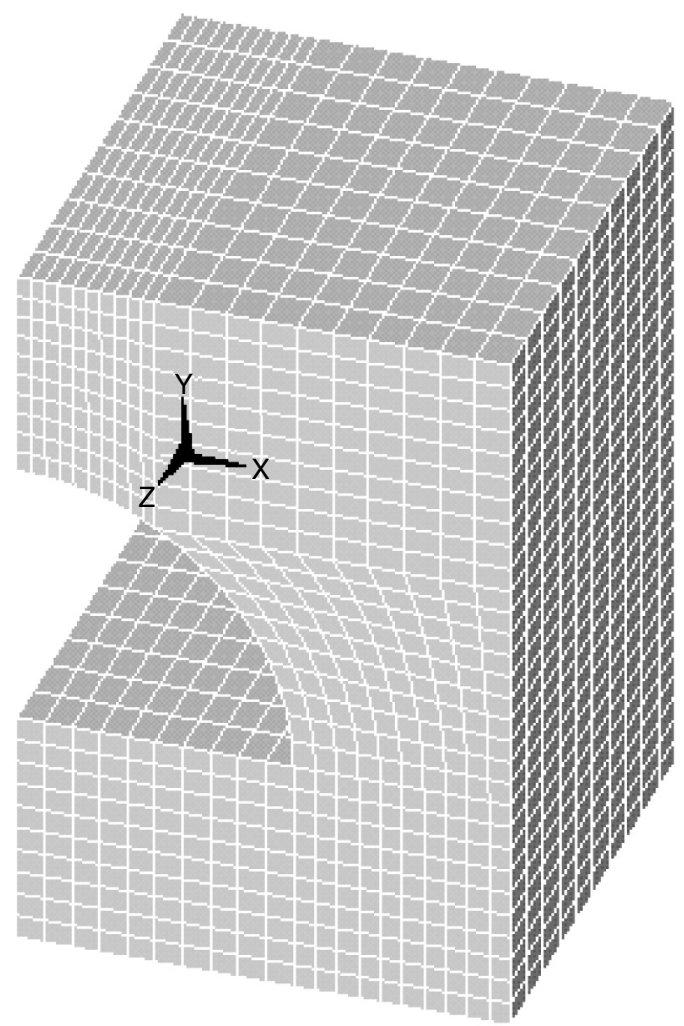

Figure 4 Finite element model. 


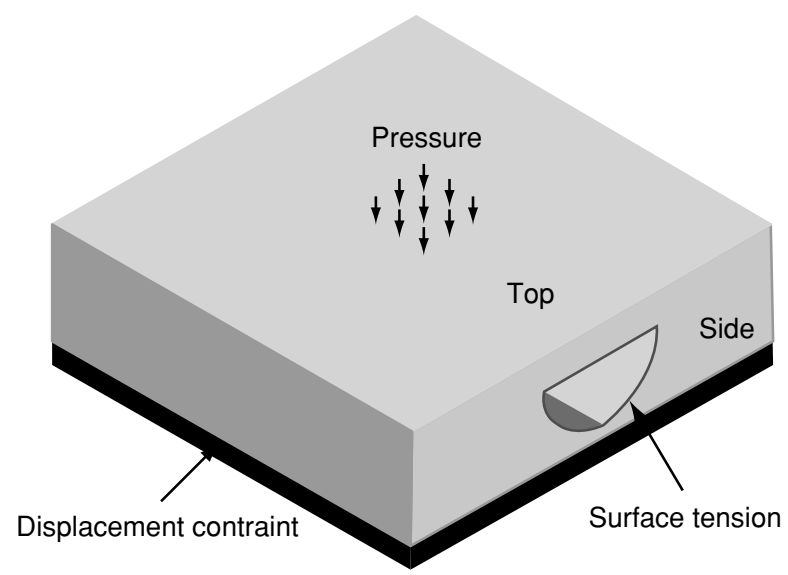

Figure 5 Loads and applied boundary conditions.

Due to the symmetric nature of problem half part of model was simulated. Symmetry boundary conditions were applied on $\mathrm{y}-\mathrm{z}$ planer side of the model to save time and computational resources.

\subsection{BOUNDARY CONDITIONS}

Commercially available FEM software ANSYS v11.0 $[8,9,10]$ has been used. The model consists of a block of material, with the channel in the centre as shown in figure 5. The crosssectional area of the valve was kept as constant and equal to $5000 \mu \mathrm{m}^{2}$ for each shape analyzed.

Figure 3 describes the loads and boundary conditions which were simulated. A constant pressure was applied over the top surface of the block of material, whilst the bottom surface was constrained to have zero displacement. Pressure due to fluid moving through the channel is applied as surface tension in the inner wall of the channel, it was computed using Laplace relation as suggested in Zhongbing Huang et al [14]. Four different cross-sectional shapes were considered. Table 1 summarizes the different cross-sectional shapes which were modeled; the results obtained under the application of constant pressure of $10 \mathrm{KPa}$.

Due to the unsymmetrical nature of semi-circular channel (not symmetrical against horizontal plane) pressure load was applied in 2 different configurations. Table 1, shows the displacement of each cross-sectional shape with application of constant pressure. SemiCircular Channel (B) shape results in maximum deformation caused by applying the same normal pressure, to each of the valve shapes; as can be seen the semi-circular channel (B) appears to give the most favorable results. However, it can also be seen that this shape also results in one of the highest local stresses, which could cause problems. Thus, the valve based on the semi-circular channel was selected for further optimization. The geometric parameters which can be varied for the semi-circular valve (shown in detail in figure 6) include:

- ' $w$ ' = width of channel

- ' $h$ ' = height of channel

- ' $\mathrm{t}$ ' = thickness of PDMS membrane between channel and pressure load

Of the above parameters the effect of varying the thickness of membrane between the load and channel ' $t$ ', and height of channel ' $h$ ' were studied in detail. In all cases the crosssectional area was kept constant, and 'L1' and 'L2' were fixed. The minimum possible thickness $[2,4]$ that can be manufactured is $10 \mu \mathrm{m}$, so, 't' was varied from 10 to $30 \mu \mathrm{m}$. If ' $\mathrm{h}$ ' is made too small, the flow will be restricted even when the valve is open; hence, ' $h$ ' was 
Table 1 Different cross-sectional shapes and results

\begin{tabular}{lll} 
Shape & Cross-Sectional Shape & $\begin{array}{l}\text { FEM Results under the } \\
\text { action of equal load }\end{array}$ \\
\hline $\begin{array}{l}\text { Box-Shape } \\
\text { Channel } \\
\text { (Standard) }\end{array}$ & $\begin{array}{l}\text { Maximum Displacement }=\alpha \\
\text { Maximum Stress }=\beta\end{array}$ \\
& & \\
\end{tabular}

$\begin{aligned} & \text { Eye-Shape } \\ & \text { Channel }\end{aligned}$
$\begin{aligned} & \text { Maximum Displacement }=0.90 \alpha \\ & \text { Maximum Stress }=0.80 \beta\end{aligned}$
$\begin{aligned} & \text { Chircular } \\ & \text { Channel } \\ & \text { Circular } \\ & \text { Channel } \\ & \text { (B)* }\end{aligned}$
$\begin{aligned} & \text { Maximum Displacement }=1.18 \alpha \\ & \text { Maximum Stress }=1.48 \beta\end{aligned}$
$\begin{aligned} & \text { Maximum Displacement }=1.32 \alpha \\ & \text { Channel }\end{aligned}$

always larger than $40 \mu \mathrm{m}$ [1]. As cross-sectional area of channel kept as constant throughout so values of ' $h$ ' and ' $w$ ' are numerically dependent. Table 2 shows the different configurations which were simulated.

\section{RESULTS AND DISCUSSION}

Contour plots of displacement and stress for each of the configurations in table 2 are given in table 3, for the pressure loading until valve closes or pressure load reaches to $10 \mathrm{KPa}$.

The pressure required to close the valve also varied between configurations. Figure 8 shows the pressure required to close the valve, again as a function of ' $h$ ' and ' $t$ '.

Figure 7 clearly shows that that the valve with minimum thickness and minimum height requires the least applied pressure to close. This is as expected. Also marked on figure 7 is a region where the valve does not close even if the magnitude of the applied pressure reaches $10 \mathrm{KPa}$. (i.e. the malfunction region).

One important result from the simulation is the maximum local stress, since a high stress could result in mechanical failure. Figure 8 shows the maximum stress in each of the 

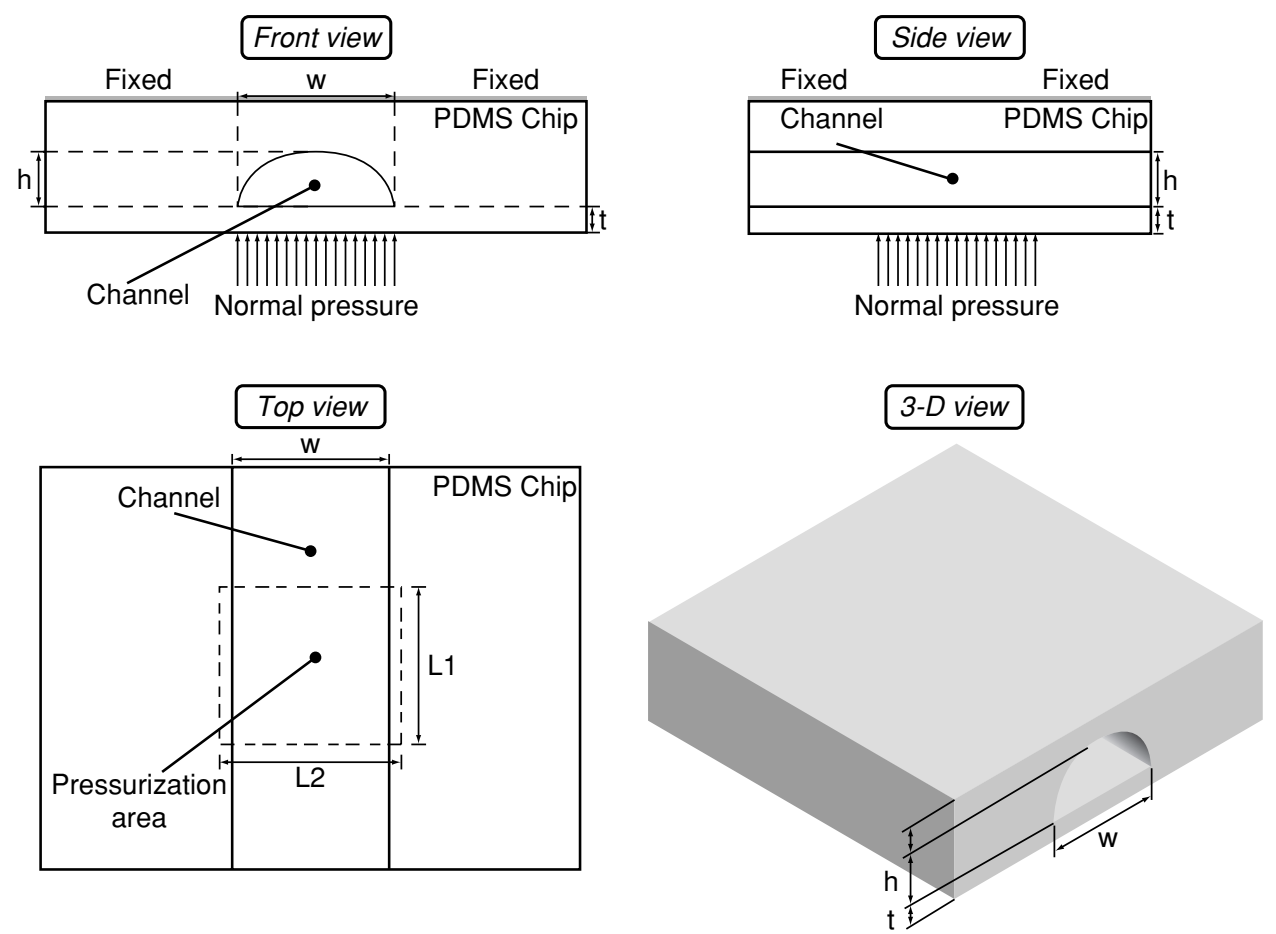

Figure 64 Views of selected channel configuration.

Table 2 Multiple configurations for ' $h$ ' and 't' parameters

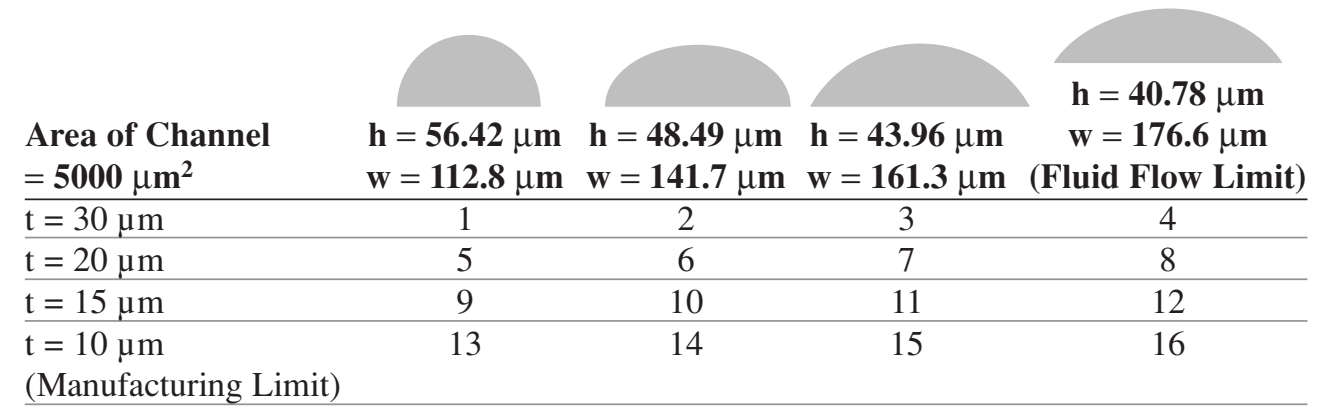


Table 3 Von-Mises stress \& displacement contours of 16 configurations

Config. Von-Mises Stress Contour Plot (KPa) Displacement Contour Plot (mm)

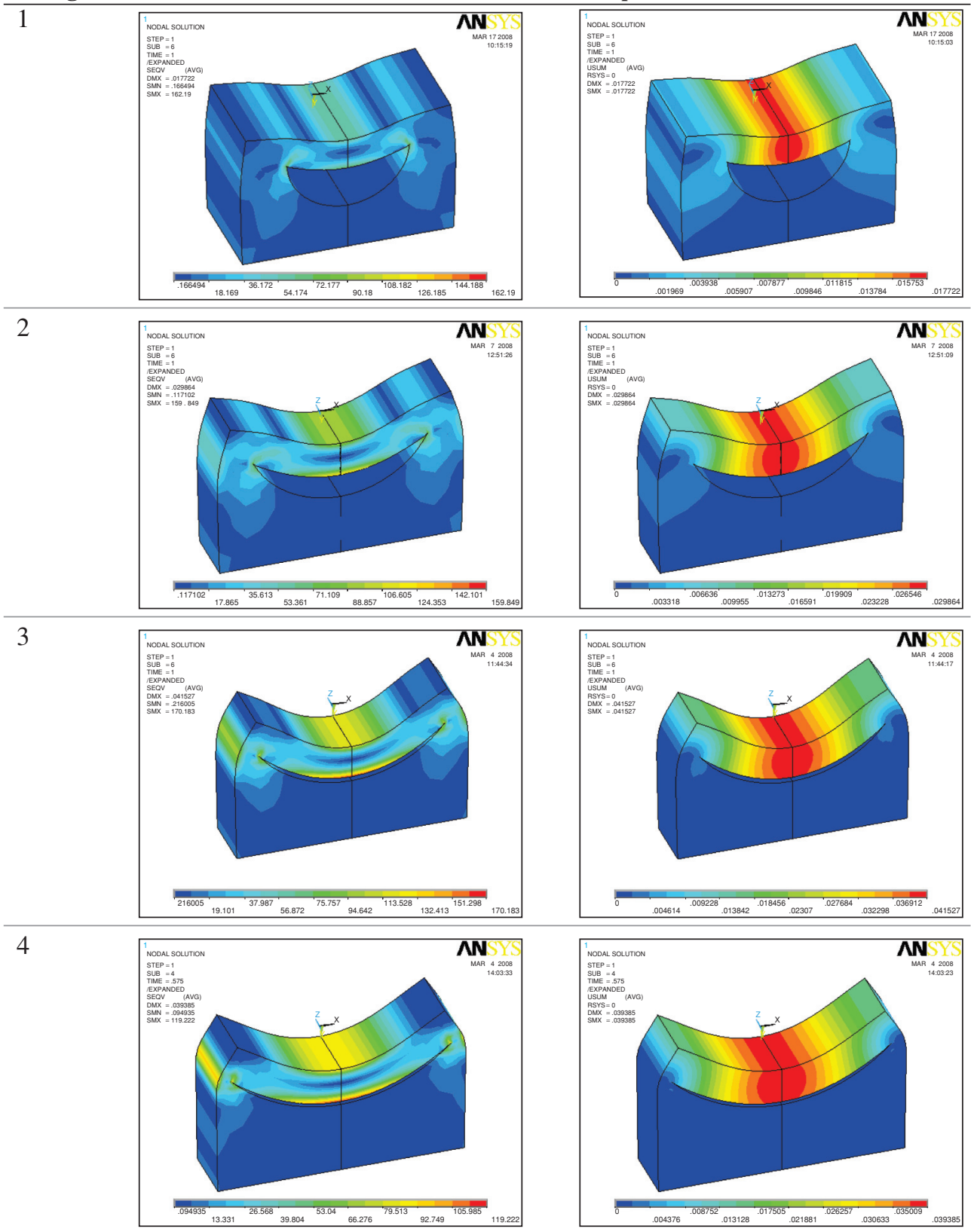


Table 3 (continued)

Config. Von-Mises Stress Contour Plot (KPa)

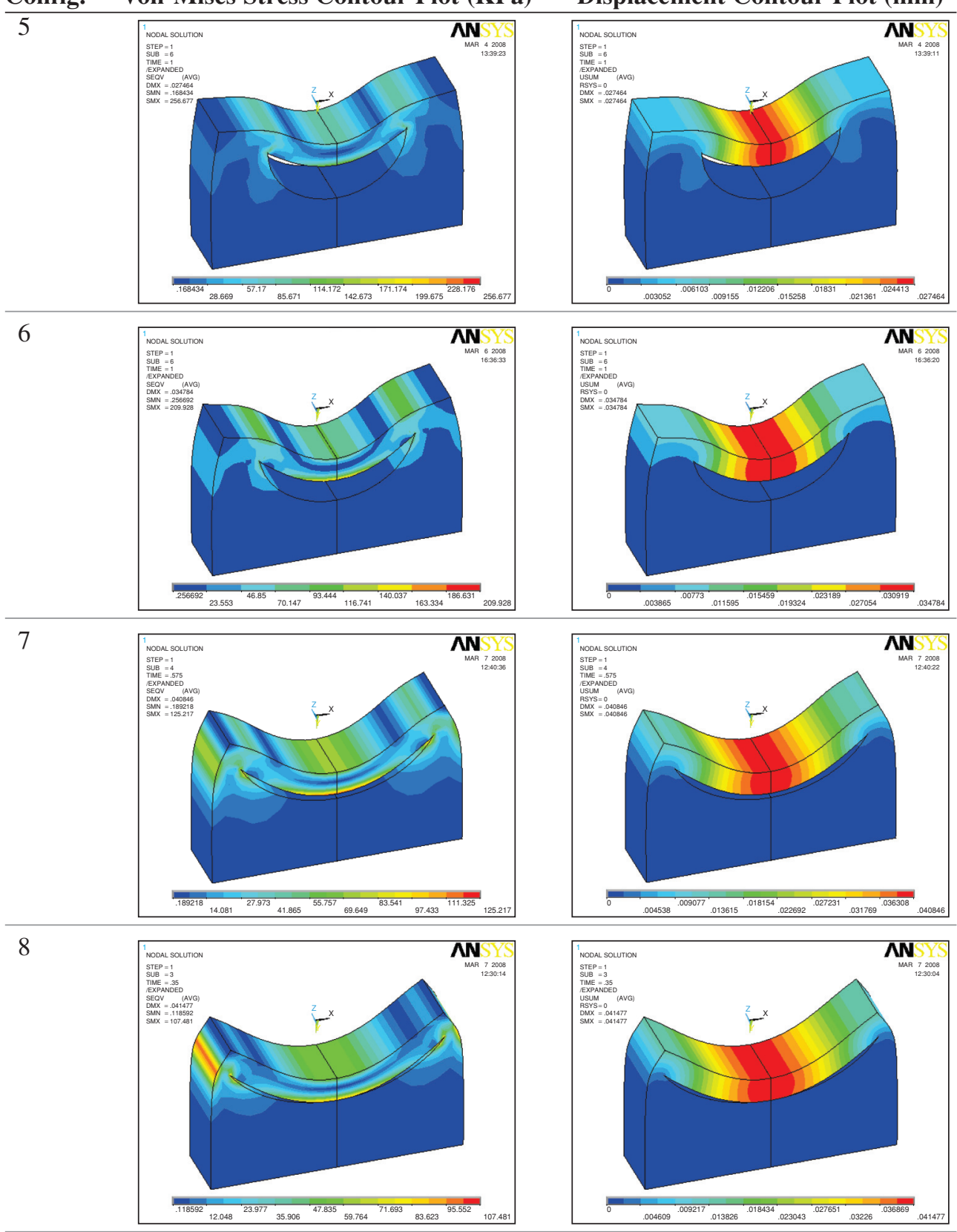

Displacement Contour Plot (mm) 
Table 3 (continued)

Config. Von-Mises Stress Contour Plot (KPa) Displacement Contour Plot (mm)

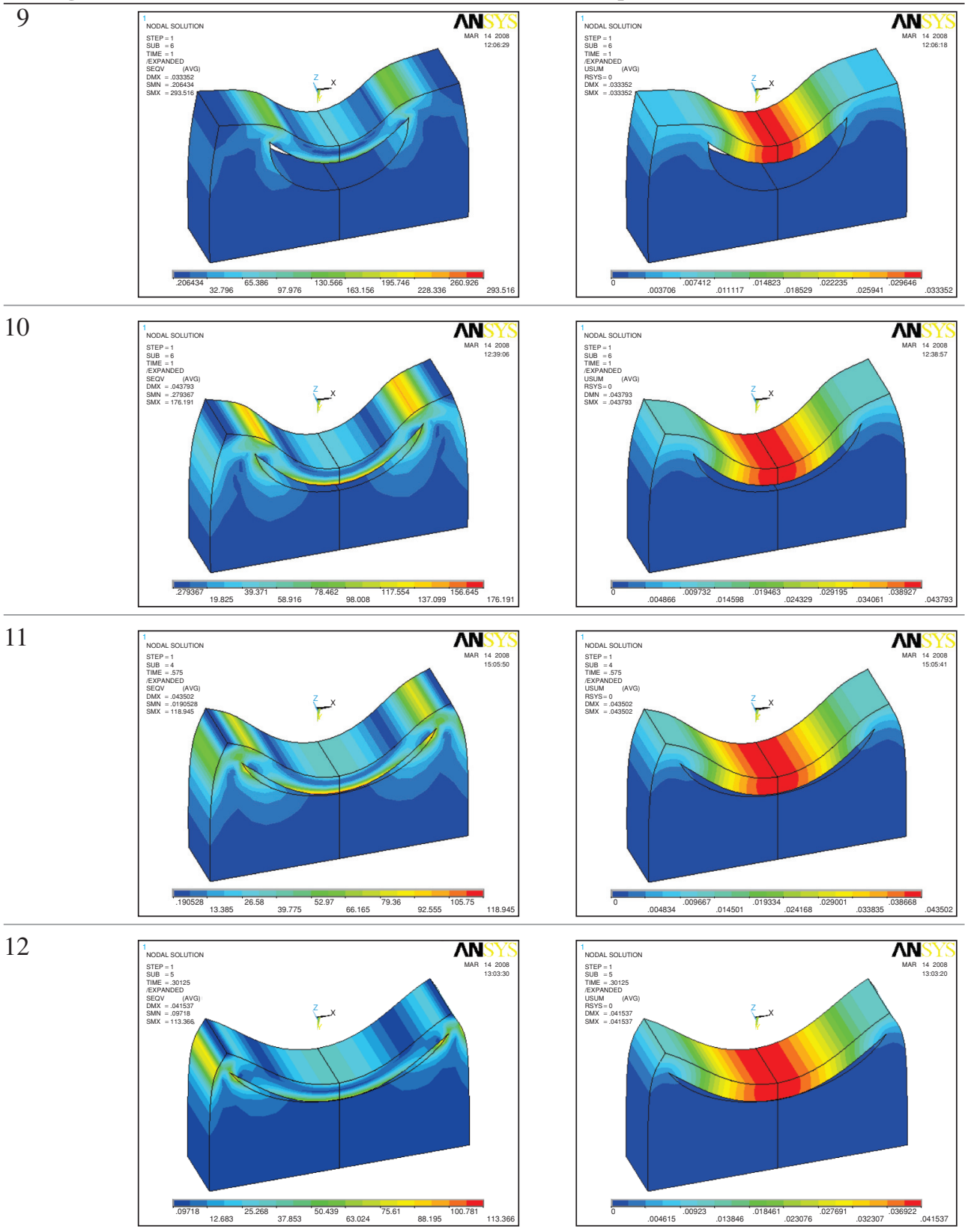


Table 3 (continued)

Config. Von-Mises Stress Contour Plot (KPa)

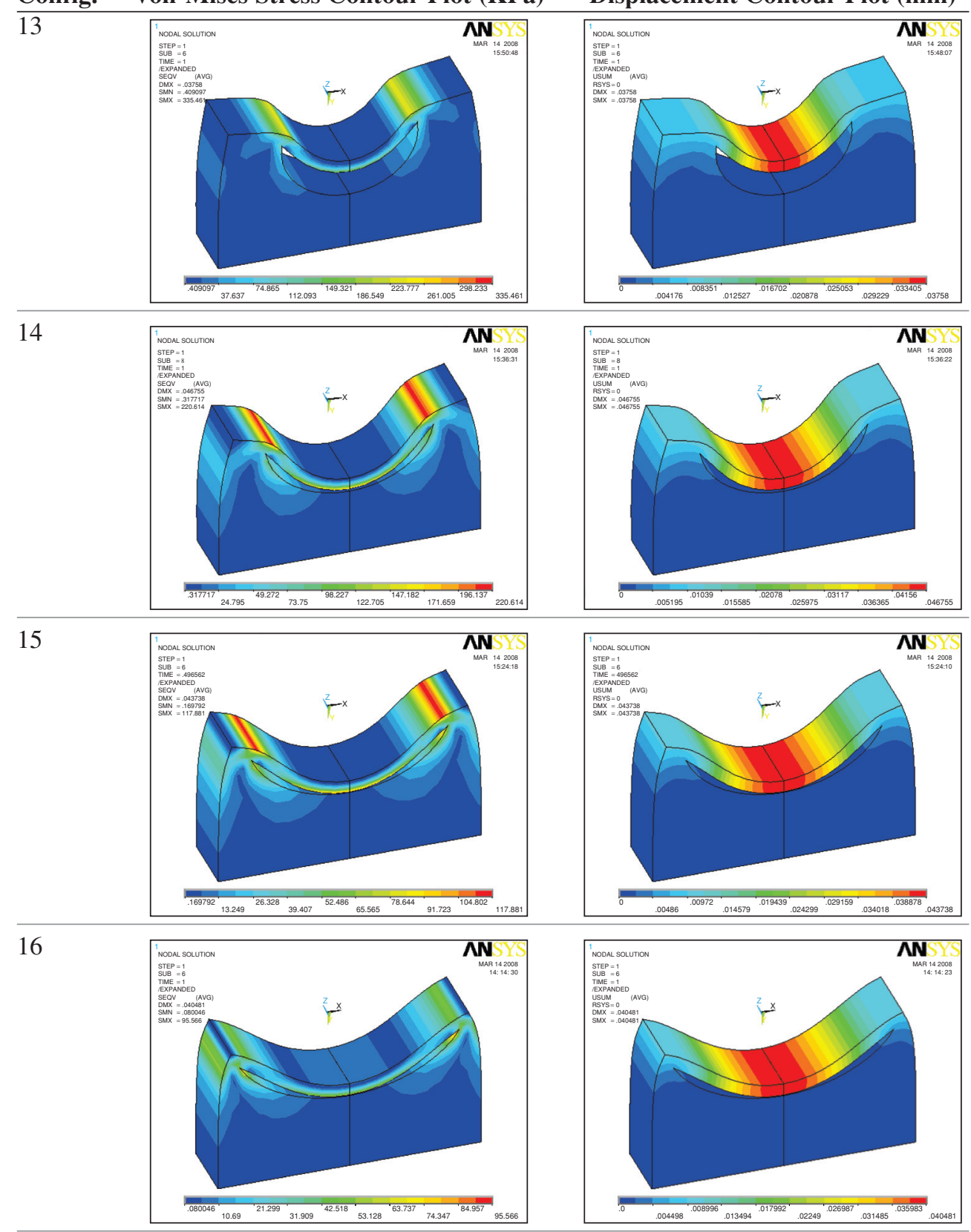

Displacement Contour Plot (mm) 


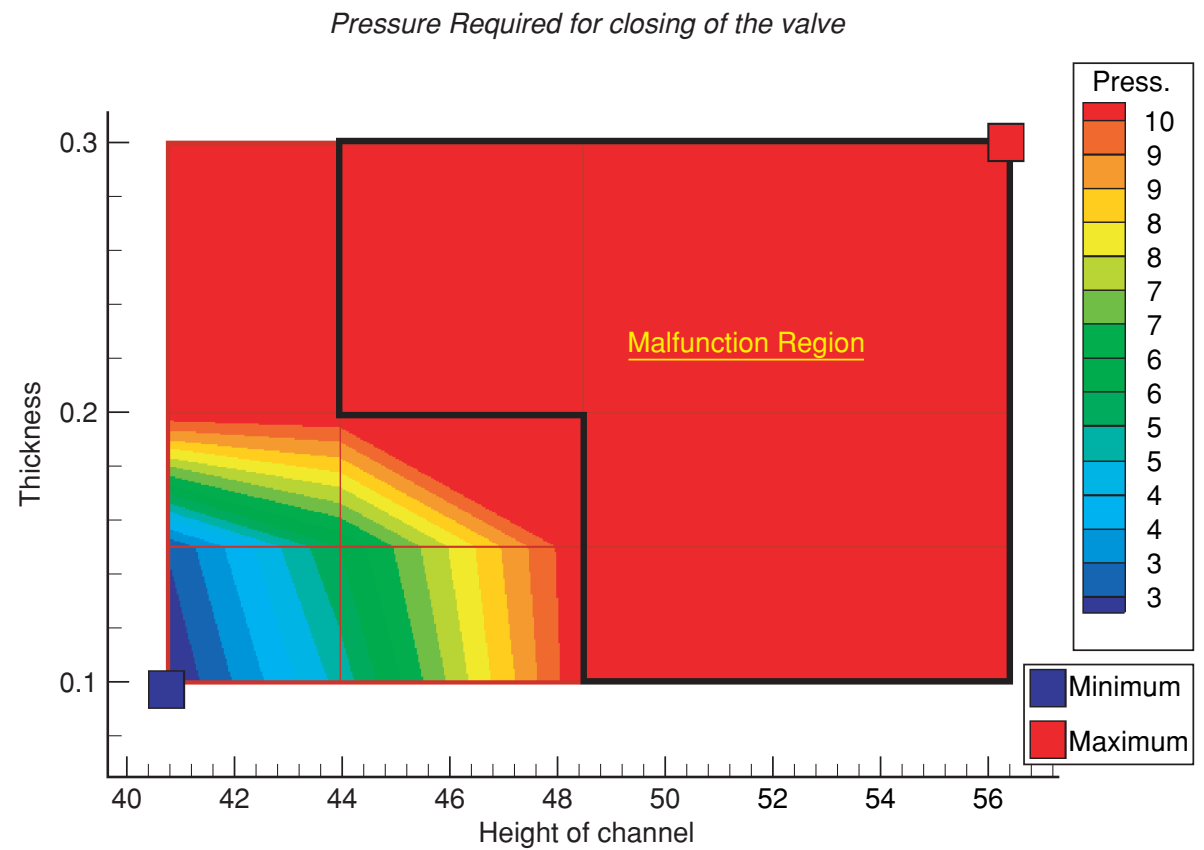

Figure 7 Contour plot of pressure $(\mathrm{KPa})$ requirement variation with thickness $(\mathrm{t})$ and height (h) Parameters.

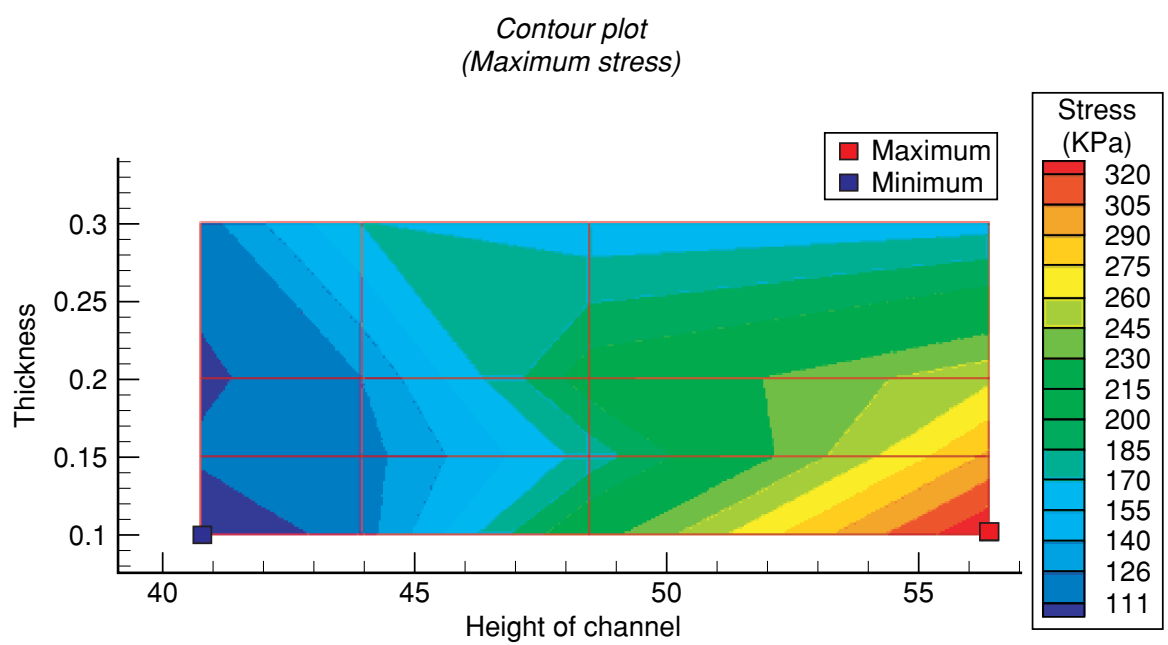

Figure 8 Contour plot of maximum von-mises stress (KPa) variation with thickness (t) and height $(h)$ parameters.

simulations, as a function of $\mathrm{h}$ and $\mathrm{t}$. It is to be noted that we are not considering diffusion across PDMS. This assumption is particularly good if we use liquid to apply pressure.

As shown in figure 8, the maximum stress occurs when the thickness is at minimum and height of channel is at a maximum (i.e. configuration 13 in table 2). The configuration which gave the minimum stress had the lowest value of $\mathrm{h}$ and the lowest value of $\mathrm{t}$ (configuration 16 
in table 2). However, it should be noted that at low values of $h$, the thickness, $t$, has only a weak influence on the maximum local stress.

\section{CONCLUSION}

Micro fluidic valve is modeled and analyzed using finite element methods. Optimization resulted in a configuration that is most suitable as per its operation as valve in microfluidic chip. It was also found out that optimized configuration is on the verge of fluid dynamic and manufacturing constraints. Moreover trend to performance of each dimensional parameter is known (Figure 7 and Figure 8), based on which optimization can be achieved for other application of microfludic valves.

\section{ACKNOWLEDGMENT}

Authors acknowledge the support of Dr. Stuart Scott, Lecturer in Department of Engineering, University of Cambridge for proof reading and helpful suggestions.

\section{REFERENCES}

[1] Kangsun Lee et al, Fabrication of round channels using the surface tension of PDMS and its application to a 3D serpentine mixer, Journal of Micromechanics and Microengineering, 2007, $17,1533-1541$.

[2] Kurt Miller, Testing Elastomers for Hyperelastic Material Models in Finite Element Analysis, Axel Products ${ }^{\circledR}$ Inc., June 2004, V3.

[3] Marc A. Unger et al, Monolithic Microfabricated Valves and Pumps by Multilayer Soft Lithography, Science, 2000, 288 (113), DOI: 10.1126/science.288.5463.113

[4] R. C. Huang and L. Anand, Non-linear mechanical behavior of the elastomer polydimethylsiloxane (PDMS) used in the manufacture of microfluidic devices, to be published

[5] R. Quake et al, Experimentally validated quantitative linear model for the device physics of elastomeric microfluidic valves, Journal of Applied Physics, 2007, 10 (064505), DOI: $10.1063 / 1.2511688$

[6] J C Lotters et al, The mechanical properties of the rubber elastic polymer polydimethylsiloxane for sensor applications, Journal of Micromechanics and Microengineering, 1997, 7, 145-147.

[7] Mark Lee Adams, Integration of Optoelectronics and Microfluidics for biological and chemical sensing, PhD, California Institute of Technology, 2003.

[8] Multiphysics Software, ANSYS ${ }^{\circledR}$ Inc. Version 11.0.

[9] Theory Reference, ${ }^{1}$ Structures, ${ }^{2}$ Hyperelastic Material Models, ${ }^{3}$ Non-Linear Analysis, ANSYS ${ }^{\circledR}$ Inc. Version 11.0.

[10] Documentation, ${ }^{1}$ Structural Analysis Guide, and ${ }^{2}$ Verification Manuals, ANSYS ${ }^{\circledR}$ Inc. Release 11.0

[11] Mario $\mathrm{M}$ et al, Hyperelastic constitutive modeling under finite strain, International Journal of Solids and Structures, 2004, 41, 5327-5350.

[12] E. Cosola et al, A general framework for identification of hyper-elastic membranes with moiré techniques and multi-point simulated annealing, International Journal of Solids and Structures, 2008, 45, 6074-6099.

[13] Ordieres-Meré a et al, Finite element analysis of the hyper-elastic contact problem in automotive door sealing, Journal of Non-Crystalline Solids, 2008, 354, 5331-5333.

[14] Zhongbing Huang et al, Equilibrium of drops on inclined fibers, Journal of Colloid and Interface Science 2008, 330 (2009), 399-403. 